\title{
Simulating a Quantum Harmonic Oscillator by introducing it To a Bosonic System
}

\author{
A Preprint \\ Rajdeep Tah ${ }^{1 *}$ \\ ${ }^{1}$ School of Physical Sciences \\ ${ }^{1}$ National Institute of Science Education and Research, Bhubaneswar, HBNI, P.O. Jatni, Khurda-752050, \\ Odisha, India.
}

August 22, 2020

\begin{abstract}
The goal of this paper is to associate a Quantum Harmonic Oscillator to a Bosonic System, try to simulate it in IBMQ-Experience (at 8192 shots) and further study it. We associated the concept of Pauli Matrix equivalent to Bosonic Particles and used it to calculate the Unitary Operators which helped us to theoretically visualize each Quantum states and further simulate our system.
\end{abstract}

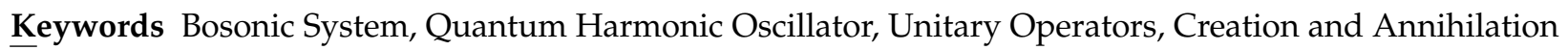
operators

\section{INTRODUCTION}

Harmonic oscillator is one of the most fundamental problems in the field of Physics and it is involved in all aspects of Physics. The reason is still unknown to us but it is very natural for us to understand that whenever a system is disturbed from its minimum energy state then in the course of attaining minimum energy state again, the system will tend to oscillate. This is how a harmonic oscillator functions in a classical sense. Hence, it is worth to search for such a system in the quantum world, too. Thus, Quantum Harmonic Oscillator[1] is nothing but a quantum mechanical analog of the classical harmonic oscillator.

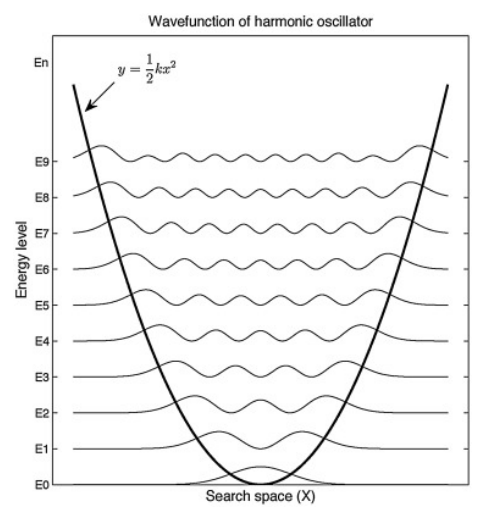

Figure 1: A Generalized Representation of Quantum Harmonic Oscillator [2]

\footnotetext{
*rajdeep.tah@niser.ac.in
} 
A Quantum Harmonic Oscillator is different from a Classical Harmonic Oscillator mainly on the basis of three grounds: First, the ground energy state for a quantum harmonic oscillator is non-zero because there exists fluctuations as a result of Heisenberg Uncertainty Principle[3]: Second, a particle in a quantum harmonic oscillator potential can be found outside the region $-\mathrm{A} \leq x \leq+\mathrm{A}$ with a non-zero probability: Thirdly, the probability density distributions for a quantum oscillator in the ground low-energy state is largest at the middle of the well[4]. It is commonly used as a model to study the vibrations of the atomic particles and molecules under the effect of classical spring like potential which is a commonly accepted model for the molecular bonding. QHO (quantum harmonic oscillator) is one of the exactly solvable models in the field of quantum mechanics having solutions in the form of Hermite polynomials and it can be generalized to $\mathrm{N}$-dimensions[5]. Its application is not only restricted to the study of simple di-atomic molecule, but it's in fact expanded to the different domains of Physics, e.g. in the study of complex modes of vibration in larger molecule, the theory of heat capacity, QHO as a thermodynamic heat engine[6], etc.

\section{MATHEMATICAL MODELLING}

We can derive the Pauli Matrix Equivalents for Bosonic Systems by using the following three equations[7]:

$$
\begin{gathered}
\left(\sigma_{3}\right)_{j l}=\frac{\left\langle s, j\left|S_{k}\right| s, l\right\rangle}{s \hbar}=\frac{j}{s} \delta_{i j} \\
\left(\sigma_{1}\right)_{j l}=\frac{[s(s+1)-j(j-1)]^{1 / 2}}{2 s} \delta_{j l+1}+\frac{[s(s+1)-j(j+1)]^{1 / 2}}{2 s} \delta_{j l-1} \\
\left(\sigma_{2}\right)_{j l}=\frac{[s(s+1)-j(j-1)]^{1 / 2}}{2 i s} \delta_{j l+1}-\frac{[s(s+1)-j(j+1)]^{1 / 2}}{2 i s} \delta_{j l-1}
\end{gathered}
$$

$\therefore$ By using the above three equations, we have:

$$
\begin{gathered}
\sigma_{1}=\frac{1}{\sqrt{2}}\left[\begin{array}{lll}
0 & 1 & 0 \\
1 & 0 & 1 \\
0 & 1 & 0
\end{array}\right] \\
\sigma_{2}=\frac{1}{\sqrt{2}}\left[\begin{array}{ccc}
0 & -i & 0 \\
i & 0 & -i \\
0 & i & 0
\end{array}\right] \\
\sigma_{3}=\left[\begin{array}{ccc}
1 & 0 & 0 \\
0 & 0 & 0 \\
0 & 0 & -1
\end{array}\right]
\end{gathered}
$$

Where, $\sigma_{1}, \sigma_{2}, \sigma_{3}$ are the Pauli Matrix equivalents for Bosonic particles.

\section{HARMONIC OSCILLATOR IN BRIEF}

The most common and familiar version of the Hamiltonian of the Quantum Harmonic Oscillator in general can be written as:

$$
\hat{H}=\frac{\hat{p}^{2}}{2 m}+\frac{1}{2} m \omega^{2} \hat{x}^{2}=\frac{\hat{p}^{2}}{2 m}+\frac{1}{2} k \hat{x}^{2}
$$

where $\hat{H}$ is the Hamiltonian of the System, $\mathrm{m}$ is the mass of the particle, $\mathrm{k}$ is the bond stiffness (which is analogous to spring constant in classical mechanics), $\hat{x}$ is the position operator and $\hat{p}=-i \hbar \frac{\partial}{\partial x}$ is the momentum operator (where $\hbar$ is the reduced Plank's constant).

The analytical solution of the Schrodinger wave equation is given by Ref.[8]:

$$
\Psi=\sum_{n_{x}=0}^{\infty} \sum_{n_{y}=0}^{\infty} \frac{1}{2^{n} n !}\left(\frac{m \omega}{\pi \hbar}\right)^{1 / 2} e^{-\frac{\zeta^{2}}{2}} e^{-\frac{\beta^{2}}{2}} H_{n_{x}}(\zeta) H_{n_{y}}(\beta) U(t)
$$

Where;

$$
\zeta=\sqrt{\frac{m \omega}{\hbar}} x \text { and } \beta=\sqrt{\frac{m \omega}{\hbar}} y
$$


Here $H_{n}$ is the nth order Hermite polynomial. $\mathrm{U}(\mathrm{t})$ is the Unitary Operator of the system showing its time evolution and is given by:

$$
U(t)=\exp \left(\frac{-i t E_{n}}{\hbar}\right)=e^{\frac{-i t E_{n}}{\hbar}}
$$

Where $E_{n}$ are the allowed energy eigenvalues of the particle and are given by:

$$
E_{n}=\left(n_{x}+\frac{1}{2}\right) \hbar \omega+\left(n_{y}+\frac{1}{2}\right) \hbar \omega=\left(n_{x}+n_{y}+1\right) \hbar \omega
$$

And the states corresponding to the various energy eigenvalues are orthogonal to each other and satisfy:

$$
\int_{-\infty}^{+\infty} \psi_{j} \psi_{x} d x_{i}=0: \forall x_{i}
$$

A much simpler approach to the harmonic oscillator problem lies in the use of ladder operator method where we make use of ladder operators i.e. the creation and annihilation operators $\left(\hat{b}^{+}, \hat{b}\right)$, to find the solution of the problem.

Here $\hat{b}^{+}$denotes the 'Creation' operator and $\hat{b}$ denotes the 'Annihilation' operator in Bosonic System. We can also the Hamiltonian in terms of the creation and annihilation operators $\left(\hat{b}^{\dagger}, \hat{b}\right)[9]$ :

$$
\hat{H}=\hbar \omega\left(\hat{b} \hat{b}^{\dagger}-\frac{1}{2}\right)=\hbar \omega\left(\hat{b} \hat{b}^{+}+\frac{1}{2}\right)
$$

Now the Hamiltonian for "a discrete quantum harmonic oscillator" is given by:

$$
\hat{H}=\frac{\left(\hat{p}^{d}\right)^{2}}{2}+\frac{\left(\hat{x}^{d}\right)^{2}+\left(\hat{y}^{d}\right)^{2}}{2}
$$

Where $\hat{p}^{d}$ is the discrete momentum operator and $\hat{x}^{d}$ and $\hat{y}^{d}$ are the discrete position operators in in $\mathrm{x}$ and $\mathrm{y}$ spatial dimension respectively. Also $\hat{p}^{d}$ can be expressed as:

$$
\hat{p}^{d}=\left(F^{d}\right)^{-1} \cdot \hat{x}^{d} \cdot\left(F^{d}\right)
$$

Where $F^{d}$ is the standard discrete Quantum Fourier Transform matrix[10].

\section{UNITARY OPERATOR TRANSFORMATION}

For the sake of reducing mathematical complexity, let us assume $\hbar, \omega$ and $\mathrm{m}$ is unity. So, we can write the Schrodinger equation as:

$$
i \frac{\partial \Psi}{\partial t}=\hat{H} \Psi
$$

Which further implies:

$$
\Psi(t)=\Psi(0) \exp (-i \hat{H} t)
$$

From the above, it is vivid that the Unitary Operator to be computed is $U(t)=\exp \left(-i \hat{H}^{d} t\right)$ where $\hat{H}^{d}$ is the discretized Hamiltonian operator. So. the Unitary Operator is given by:

$$
U(t)=\exp \left(-i t\left(\frac{\left(\hat{p}^{d}\right)^{2}}{2}+\frac{\left(\hat{x}^{d}\right)^{2}+\left(\hat{y}^{d}\right)^{2}}{2}\right)\right)
$$

Or if we consider the $\mathrm{X}$-dimension only, then we get the Unitary Operator as:

$$
\left.U_{\hat{x}}(t)=\exp \left(\frac{-i t}{2}\left(\left(F^{d}\right)^{-1} \cdot \hat{(} x^{d}\right)^{2} \cdot\left(F^{d}\right)+\left(\hat{x}^{d}\right)^{2}\right)\right)
$$

Due to the discretization of space; the position operator $\left[\hat{x}^{d}\right]$, being a diagonal matrix, can be expanded by using the concept of Matrix exponential as Ref.[11]:

$$
\exp \left(-\frac{i t}{2}[A]\right)=\mathbb{I}+\sum_{m=1}^{\infty}\left(-\frac{i t}{2}\right)^{m} \frac{[A]^{m}}{m !}
$$

Here A is the corresponding Operator Matrix. 


\section{IMPLEMENTATION ON A BOSONIC SYSTEM}

The Hamiltonian of the full system is given by[12]:

$$
\hat{H}=\hat{H}_{\text {field }}+\hat{H}_{\text {atom }}+\hat{H}_{\text {int }}
$$

where $\hat{H}_{\text {field }}$ is the free Hamiltonian, $\hat{H}_{\text {atom }}$ is the atomic excitation Hamiltonian and $\hat{H}_{\text {int }}$ is the interaction Hamiltonian.

\subsection{MODEL}

We have modeled our system using Rabi Hamiltonian[13]. However, in our case we will be using somewhat modified version of Rabi Hamiltonian[14]:

$$
H_{s}=\sum_{k=1}^{2} \omega_{k} b_{k}^{\dagger} b_{k}+\frac{\omega_{0}}{2} \sigma_{3}+\sum_{k=1}^{2} g_{k}\left(e^{i \theta_{k}} b_{k}+e^{-i \theta_{k}} b_{k}^{\dagger}\right) \sigma_{1}
$$

Where $\omega_{0}$ is the frequency of the main oscillator, $\omega_{k}$ is the frequency of the k-th environment oscillator; $b_{k}^{+}$ and $b_{k}$ are the creation and annihilation operators of the main system and the k-th environmental oscillator respectively. Whereas $g_{k}$ 's are the coupling constant for the interaction between the k-th environment oscillator and the main quantum oscillator. We set $\mathrm{k}=1$ from now to prevent us from complicating the process.

For simplicity, we will consider the simplest case of our model and substitute $\mathrm{k}=1$ in our original Hamiltonian [in Eq.(18)] to obtain the special case of our Hamiltonian which will be our working Hamiltonian from now:

$$
H=\omega_{1} b_{1}^{\dagger} b_{1}+\frac{\omega_{0}}{2} \sigma_{3}+g_{1}\left(e^{i \theta_{1}} b_{1}+e^{-i \theta_{1}} b_{1}^{\dagger}\right) \sigma_{1}
$$

For simplicity we will drop the sub-script 1 from our Hamiltonian and obtain:

$$
H=\omega b^{\dagger} b+\frac{\omega_{0}}{2} \sigma_{3}+g\left(e^{i \theta} b+e^{-i \theta} b^{\dagger}\right) \sigma_{1}
$$

\subsection{RELEVANT TRANSFORMATION AND GENERALIZATION}

Now, as our system involves Bosonic particles, so the following commutation relations uphold:

$$
\begin{gathered}
{\left[b_{i}, b_{j}^{\dagger}\right] \equiv b_{i} b_{j}^{\dagger}-b_{j}^{\dagger} b_{i}=\delta_{i j}} \\
{\left[b_{i}^{\dagger}, b_{j}^{\dagger}\right]=\left[b_{i}, b_{j}\right]=0}
\end{gathered}
$$

Here $\delta_{i j}$ is known as 'Kronecker delta'.

The operators used in the Hamiltonian can be transformed according to Holstein-Primakoff transformations[15] (i.e. it maps spin operators for a system of spin-S moments on a lattice to creation and annihilation operators) as:

$$
\begin{aligned}
& \hat{S}_{j}^{+}=\sqrt{\left(2 S-\hat{n}_{j}\right)} \hat{b}_{j} \\
& \hat{S}_{j}^{-}=\hat{b}_{j}^{+} \sqrt{\left(2 S-\hat{n}_{j}\right)}
\end{aligned}
$$

where $\hat{b}_{j}^{+}\left(\hat{b}_{j}\right)$ is the creation (annihilation) operator at site $j$ that satisfies the commutation relations mentioned above and $\hat{n}_{j}=\hat{b}_{j}^{\dagger} \hat{b}_{j}$ is the "Number Operator". Hence we can generalize the above equations as:

$$
\begin{aligned}
& S^{+}=\sqrt{\left(2 S-b^{+} b\right) b} \\
& S^{-}=b^{+} \sqrt{\left(2 S-b^{+} b\right)}
\end{aligned}
$$

Where;

$$
S_{+} \equiv S_{x}+i S_{y} \quad \text { and } \quad S_{-} \equiv S_{x}-i S_{y}
$$


Where; $S_{x}\left(=\sigma_{1}\right), S_{y}\left(=\sigma_{2}\right), S_{z}\left(=\sigma_{3}\right)$ are the Pauli matrices for Bosonic system (as mentioned in the previous section).

Now by using the above transformations; we can write our creation and annihilation operators in terms of Matrices as:

$$
b^{+}=\left[\begin{array}{lll}
0 & 0 & 0 \\
1 & 0 & 0 \\
0 & 1 & 0
\end{array}\right] \quad \text { and } \quad b=\left[\begin{array}{lll}
0 & 1 & 0 \\
0 & 0 & 1 \\
0 & 0 & 0
\end{array}\right]
$$

Now the Hamiltonian for our coupled Quantum Harmonic Oscillator in Eq.(19) can be decomposed as:

$$
H=\omega b^{\dagger} b \otimes \mathbb{I}+\frac{\omega_{0}}{2} \mathbb{I} \otimes \sigma_{3}+g\left(e^{i \theta} b+e^{-i \theta} b^{\dagger}\right) \otimes \sigma_{1}
$$

Or the above equation can be written as:

$$
H=\omega b^{\dagger} b \otimes \mathbb{I}+\frac{\omega_{0}}{2} \mathbb{I} \otimes S_{3}+g\left(e^{i \theta} b+e^{-i \theta} b^{\dagger}\right) \otimes S_{1}
$$

Now, we will evaluate each term to simplify the expression of the Hamiltonian in the form of matrix. Here,

$$
\begin{aligned}
\omega b^{\dagger} b \otimes \mathbb{I} & =\omega\left[\begin{array}{lll}
0 & 0 & 0 \\
0 & 1 & 0 \\
0 & 0 & 1
\end{array}\right] \otimes\left[\begin{array}{lll}
1 & 0 & 0 \\
0 & 1 & 0 \\
0 & 0 & 1
\end{array}\right] \\
\Rightarrow \omega b^{\dagger} b \otimes \mathbb{I} & =\left[\begin{array}{lllllllll}
0 & 0 & 0 & 0 & 0 & 0 & 0 & 0 & 0 \\
0 & 0 & 0 & 0 & 0 & 0 & 0 & 0 & 0 \\
0 & 0 & 0 & 0 & 0 & 0 & 0 & 0 & 0 \\
0 & 0 & 0 & \omega & 0 & 0 & 0 & 0 & 0 \\
0 & 0 & 0 & 0 & \omega & 0 & 0 & 0 & 0 \\
0 & 0 & 0 & 0 & 0 & \omega & 0 & 0 & 0 \\
0 & 0 & 0 & 0 & 0 & 0 & \omega & 0 & 0 \\
0 & 0 & 0 & 0 & 0 & 0 & 0 & \omega & 0 \\
0 & 0 & 0 & 0 & 0 & 0 & 0 & 0 & \omega
\end{array}\right]
\end{aligned}
$$

Similarly,

Finally,

$$
\begin{gathered}
\frac{\omega_{0}}{2} \mathbb{I} \otimes S_{z}=\frac{\omega_{0}}{2}\left[\begin{array}{ccc}
1 & 0 & 0 \\
0 & 1 & 0 \\
0 & 0 & 1
\end{array}\right] \otimes\left[\begin{array}{ccccc}
1 & 0 & 0 \\
0 & 0 & 0 \\
0 & 0 & -1
\end{array}\right] \\
\Rightarrow \frac{\omega_{0}}{2} \mathbb{I} \otimes S_{z}=\left[\begin{array}{ccccccccc}
\frac{\omega_{0}}{2} & 0 & 0 & 0 & 0 & 0 & 0 & 0 & 0 \\
0 & 0 & 0 & 0 & 0 & 0 & 0 & 0 & 0 \\
0 & 0 & -\frac{\omega_{0}}{2} & 0 & 0 & 0 & 0 & 0 & 0 \\
0 & 0 & 0 & \frac{\omega_{0}}{2} & 0 & 0 & 0 & 0 & 0 \\
0 & 0 & 0 & 0 & 0 & 0 & 0 & 0 & 0 \\
0 & 0 & 0 & 0 & 0 & -\frac{\omega_{0}}{2} & 0 & 0 & 0 \\
0 & 0 & 0 & 0 & 0 & 0 & \frac{\omega_{0}}{2} & 0 & 0 \\
0 & 0 & 0 & 0 & 0 & 0 & 0 & 0 & 0 \\
0 & 0 & 0 & 0 & 0 & 0 & 0 & 0 & -\frac{\omega_{0}}{2}
\end{array}\right]
\end{gathered}
$$

$$
\begin{gathered}
g\left(e^{i \theta} b+e^{-i \theta} b^{\dagger}\right) \otimes S_{x}=\frac{g}{\sqrt{2}}\left[\begin{array}{cccc}
0 & e^{i \theta} & 0 \\
e^{-i \theta} & 0 & e^{i \theta} \\
0 & e^{-i \theta} & 0
\end{array}\right] \otimes\left[\begin{array}{ccc}
0 & 1 & 0 \\
1 & 0 & 1 \\
0 & 1 & 0
\end{array}\right] \\
\Rightarrow g\left(e^{i \theta} b+e^{-i \theta} b^{\dagger}\right) \otimes S_{x}=\frac{g}{\sqrt{2}}\left[\begin{array}{ccccccccc}
0 & 0 & 0 & 0 & e^{i \theta} & 0 & 0 & 0 & 0 \\
0 & 0 & 0 & e^{i \theta} & 0 & e^{i \theta} & 0 & 0 & 0 \\
0 & 0 & 0 & 0 & e^{i \theta} & 0 & 0 & 0 & 0 \\
0 & e^{-i \theta} & 0 & 0 & 0 & 0 & 0 & e^{i \theta} & 0 \\
e^{-i \theta} & 0 & e^{-i \theta} & 0 & 0 & 0 & e^{i \theta} & 0 & e^{i \theta} \\
0 & e^{-i \theta} & 0 & 0 & 0 & 0 & 0 & e^{i \theta} & 0 \\
0 & 0 & 0 & 0 & e^{-i \theta} & 0 & 0 & 0 & 0 \\
0 & 0 & 0 & e^{-i \theta} & 0 & e^{-i \theta} & 0 & 0 & 0 \\
0 & 0 & 0 & 0 & e^{-i \theta} & 0 & 0 & 0 & 0
\end{array}\right]
\end{gathered}
$$


Substituting the above values in Eq.(27), we get the value of $H$ (a $9 \times 9$ matrix) as:

$$
\Rightarrow H=\left[\begin{array}{ccccccccc}
\frac{\omega_{0}}{2} & 0 & 0 & 0 & \frac{g e^{i \theta}}{\sqrt{2}} & 0 & 0 & 0 & 0 \\
0 & 0 & 0 & \frac{g e^{i \theta}}{\sqrt{2}} & 0 & \frac{g e^{i \theta}}{\sqrt{2}} & 0 & 0 & 0 \\
0 & 0 & -\frac{\omega_{0}}{2} & 0 & \frac{g e^{i \theta}}{\sqrt{2}} & 0 & 0 & 0 & 0 \\
0 & \frac{g e^{-i \theta}}{\sqrt{2}} & 0 & \left(\omega+\frac{\omega_{0}}{2}\right) & 0 & 0 & 0 & \frac{g e^{i \theta}}{\sqrt{2}} & 0 \\
\frac{g e^{-i \theta}}{\sqrt{2}} & 0 & \frac{g e^{-i \theta}}{\sqrt{2}} & 0 & \omega & 0 & \frac{g e^{i \theta}}{\sqrt{2}} & 0 & \frac{g e^{i \theta}}{\sqrt{2}} \\
0 & \frac{g e^{-i \theta}}{\sqrt{2}} & 0 & 0 & 0 & \left(\omega-\frac{\omega_{0}}{2}\right) & 0 & \frac{g e^{i \theta}}{\sqrt{2}} & 0 \\
0 & 0 & 0 & 0 & \frac{g e^{-i \theta}}{\sqrt{2}} & 0 & \left(\omega+\frac{\omega_{0}}{2}\right) & 0 & 0 \\
0 & 0 & 0 & \frac{g e^{-i \theta}}{\sqrt{2}} & 0 & \frac{g e^{-i \theta}}{\sqrt{2}} & 0 & \omega & 0 \\
0 & 0 & 0 & 0 & \frac{g e^{-i \theta}}{\sqrt{2}} & 0 & 0 & 0 & \left(\omega-\frac{\omega_{0}}{2}\right)
\end{array}\right]
$$

\section{DERIVATION OF UNITARY OPERATORS}

Clearly, we know that for a system with Hamiltonian $H$, the unitary operator is given by:

$$
U=e^{-i H t}
$$

Where $H$ is the Hamiltonian of the system derived in the previous section.

But to find the unitary operator compatible, we need to change the form of our Hamiltonian and write it as a sum of two matrices whose corresponding unitary operators are relatively easier to compute:

$$
H=X+Y
$$

Where,

$$
\begin{aligned}
X & =\left[\begin{array}{ccccccccc}
\frac{\omega_{0}}{2} & 0 & 0 & 0 & 0 & 0 & 0 & 0 & 0 \\
0 & 0 & 0 & 0 & 0 & 0 & 0 & 0 & 0 \\
0 & 0 & -\frac{\omega_{0}}{2} & 0 & 0 & 0 & 0 & 0 & 0 \\
0 & 0 & 0 & \left(\omega+\frac{\omega_{0}}{2}\right) & 0 & 0 & 0 & 0 & 0 \\
0 & 0 & 0 & 0 & \omega & 0 & 0 & 0 & 0 \\
0 & 0 & 0 & 0 & 0 & \left(\omega-\frac{\omega_{0}}{2}\right) & 0 & 0 & 0 \\
0 & 0 & 0 & 0 & 0 & 0 & \left(\omega+\frac{\omega_{0}}{2}\right) & 0 & 0 \\
0 & 0 & 0 & 0 & 0 & 0 & 0 & \omega & 0 \\
0 & 0 & 0 & 0 & 0 & 0 & 0 & 0 & \left(\omega-\frac{\omega_{0}}{2}\right)
\end{array}\right] \\
Y & =\left[\begin{array}{ccccccccc}
0 & 0 & 0 & 0 & \frac{g e^{i \theta}}{\sqrt{2}} & 0 & 0 & 0 & 0 \\
0 & 0 & 0 & \frac{g e^{i \theta}}{\sqrt{2}} & 0 & \frac{g e^{i \theta}}{\sqrt{2}} & 0 & 0 & 0 \\
0 & 0 & 0 & 0 & \frac{g e^{i \theta}}{\sqrt{2}} & 0 & 0 & 0 & 0 \\
0 & \frac{g e^{-i \theta}}{\sqrt{2}} & 0 & 0 & 0 & 0 & 0 & \frac{g e^{i \theta}}{\sqrt{2}} & 0 \\
\frac{g e^{-i \theta}}{\sqrt{2}} & 0 & \frac{g e^{-i \theta}}{\sqrt{2}} & 0 & 0 & 0 & \frac{g e^{i \theta}}{\sqrt{2}} & 0 & \frac{g e^{i \theta}}{\sqrt{2}} \\
0 & \frac{g e^{-i \theta}}{\sqrt{2}} & 0 & 0 & 0 & 0 & 0 & \frac{g e^{i \theta}}{\sqrt{2}} & 0 \\
0 & 0 & 0 & 0 & \frac{g e^{-i \theta}}{\sqrt{2}} & 0 & 0 & 0 & 0 \\
0 & 0 & 0 & \frac{g e^{-i \theta}}{\sqrt{2}} & 0 & \frac{g e^{-i \theta}}{\sqrt{2}} & 0 & 0 & 0 \\
0 & 0 & 0 & 0 & \frac{g e^{-i \theta}}{\sqrt{2}} & 0 & 0 & 0 & 0
\end{array}\right]
\end{aligned}
$$

Thus we have,

$$
\begin{array}{r}
U=e^{-i X t} \cdot e^{-i Y t} \\
\Longrightarrow U=U_{x}(t) \cdot U_{y}(t)
\end{array}
$$


Where $U_{x}(t)=e^{-i X t}$ and $U_{y}(t)=e^{-i Y t}$. First we will compute $U_{y}(t)$, then $U_{x}(t)$. We can see that $U_{y}(t)$ can be expanded using Taylor series of expansion of the exponential function as:

$$
\begin{gathered}
U_{y}(t)=\exp (-i t Y)=\mathbb{I}+\sum_{m=1}^{\infty}(-i t)^{m} \frac{Y^{m}}{m !} \\
\Longrightarrow U_{y}(t)=\mathbb{I}+(-i t)^{1} \frac{Y}{1 !}+(-i t)^{2} \frac{Y^{2}}{2 !}+(-i t)^{3} \frac{Y^{3}}{3 !}+(-i t)^{4} \frac{Y^{4}}{4 !}+(-i t)^{5} \frac{Y^{5}}{5 !}+\ldots . .
\end{gathered}
$$

Now, for simplicity, let us denote $\frac{g}{\sqrt{2}}=g^{\prime}$. So, we have:

$$
\Longrightarrow U_{y}(t)=\left[1+\frac{\left(-i t g^{\prime}\right)^{2}}{2 !}+\frac{\left(-i t g^{\prime}\right)^{4}}{4 !}+\ldots\right] I+\left[\frac{\left(-i t g^{\prime}\right)}{1 !}+\frac{\left(-i t g^{\prime}\right)^{3}}{3 !}+\frac{\left(-i t g^{\prime}\right)^{5}}{5 !}+\ldots\right] M
$$

Where;

$$
M=\left[\begin{array}{ccccccccc}
0 & 0 & 0 & 0 & e^{i \theta} & 0 & 0 & 0 & 0 \\
0 & 0 & 0 & e^{i \theta} & 0 & e^{i \theta} & 0 & 0 & 0 \\
0 & 0 & 0 & 0 & e^{i \theta} & 0 & 0 & 0 & 0 \\
0 & e^{-i \theta} & 0 & 0 & 0 & 0 & 0 & e^{i \theta} & 0 \\
e^{-i \theta} & 0 & e^{-i \theta} & 0 & 0 & 0 & e^{i \theta} & 0 & e^{i \theta} \\
0 & e^{-i \theta} & 0 & 0 & 0 & 0 & 0 & e^{i \theta} & 0 \\
0 & 0 & 0 & 0 & e^{-i \theta} & 0 & 0 & 0 & 0 \\
0 & 0 & 0 & e^{-i \theta} & 0 & e^{-i \theta} & 0 & 0 & 0 \\
0 & 0 & 0 & 0 & e^{-i \theta} & 0 & 0 & 0 & 0
\end{array}\right]
$$

$\left({ }^{* *}\right.$ We can observe that $\left[Y^{2}, Y^{4}, Y^{6}, \ldots.\right]$ will give Identity matrices whereas $\left[Y^{1}, Y^{3}, Y^{5}, \ldots\right]$ will give the same matrix which is given above as M. So we differentiate them in two groups.)

$$
\begin{aligned}
& \Longrightarrow U_{y}(t)=\cos g^{\prime} t I-i M \sin g^{\prime} t \\
& \Longrightarrow U_{y}(t)=\cos \frac{g t}{\sqrt{2}} \mathbb{I}-i M \sin \frac{g t}{\sqrt{2}}
\end{aligned}
$$

Now for Bosonic particles, we need to use a 4-qubit system but for implementing a 4-qubit system we must require a $16 \times 16$ matrix because any matrix of order $N \times N$ must satisfy the condition $N=2^{n}$ (where $n=$ number of qubits). But we can express the above equation in form of a $16 \times 16$ matrix (which we have shown in the next sub-section), instead of a $9 \times 9$ matrix, by adding 1 diagonally seven times and placing 0 in other positions. In our situation we need only nine of the sixteen 4-qubit states (mentioned in Table (I)) because for the other seven states we will get the same Unitary matrix as result (i.e. without any change). We will use a 4-qubit system to simulate the above system. Therefore, we first note the results we get after operating $U_{y}(t)$ on different 4-qubit states so that we can go ahead on drawing the quantum circuit for the same.

\begin{tabular}{|c|ll|}
\hline Qubit states & Results after $\mathrm{U}_{y}($ l)acts \\
\hline$|0000\rangle$ & $\left(\cos \frac{g t}{\sqrt{2}}|0000\rangle-i \sin \frac{g t}{\sqrt{2}} e^{-i \theta}|0100\rangle\right)$ & \\
\hline$|0001\rangle$ & $\left(\cos \frac{g t}{\sqrt{2}}|0001\rangle-i \sin \frac{g t}{\sqrt{2}} e^{-i \theta}(|0011\rangle+0101)\right)$ \\
\hline$|0010\rangle$ & $\left(\cos \frac{g t}{\sqrt{2}}|0010\rangle-i \sin \frac{g t}{\sqrt{2}} e^{-i \theta}|0100\rangle\right)$ & \\
\hline & $\left(\cos \frac{g t}{\sqrt{2}}|0011\rangle-i \sin \frac{g t}{\sqrt{2}} e^{-i \theta}|0001\rangle \quad\right.$ & - \\
\hline$|0011\rangle$ & $\left.i \sin \frac{g t}{\sqrt{2}} e^{i \theta}|0111\rangle\right)$ & \\
\hline & $\left(\cos \frac{g t}{\sqrt{2}}|0100\rangle-i \sin \frac{g t}{\sqrt{2}} e^{-i \theta}(|0110\rangle \quad+\right.$ \\
\hline$|0100\rangle$ & $\left.|1000\rangle)-i \sin \frac{g t}{\sqrt{2}} e^{i \theta}(|0000\rangle+|0010\rangle)\right)$ & \\
\hline & $\left(\cos \frac{g t}{\sqrt{2}}|0101\rangle-i \sin \frac{g t}{\sqrt{2}} e^{-i \theta}|0111\rangle\right.$ & - \\
\hline$|0101\rangle$ & $\left.i \sin \frac{g t}{\sqrt{2}} e^{i \theta}|0001\rangle\right)$ \\
\hline$|0110\rangle$ & $\left(\cos \frac{g t}{\sqrt{2}}|0110\rangle-i \sin \frac{g t}{\sqrt{2}} e^{i \theta}|0100\rangle\right)$ \\
\hline$|0111\rangle$ & $\left(\cos \frac{g t}{\sqrt{2}}|0111\rangle-i \sin \frac{g t}{\sqrt{2}} e^{i \theta}(|0011\rangle+0101)\right)$ \\
\hline$|1000\rangle$ & $\left(\cos \frac{g t}{\sqrt{2}}|1000\rangle-i \sin \frac{g t}{\sqrt{2}} e^{i \theta}|0100\rangle\right)$ \\
\hline
\end{tabular}

TABLE I. Operator $U_{y}(l)$ acting on Qubit States. 
Now, we need to disentangle the final Quantum states after $U_{y}(t)$ Operator acts on the Qubit states to be able to create the Quantum circuit. So, we can disentangle the final result as:

$$
\begin{gathered}
\left(\cos \frac{g t}{\sqrt{2}}|0000\rangle-\sin \frac{g t}{\sqrt{2}} e^{-i \theta}|0100\rangle\right)=|0\rangle \otimes\left(\cos \frac{g t}{\sqrt{2}}|0\rangle-\sin \frac{g t}{\sqrt{2}} e^{-i \theta}|1\rangle\right) \otimes|0\rangle \otimes|0\rangle \\
\cdot \\
\cdot \\
\left.\cdot \cos \frac{g t}{\sqrt{2}}|0110\rangle-\sin \frac{g t}{\sqrt{2}} e^{i \theta}|0100\rangle\right)=|0\rangle \otimes|1\rangle \otimes\left(\cos \frac{g t}{\sqrt{2}}|1\rangle-\sin \frac{g t}{\sqrt{2}} e^{i \theta}|0\rangle\right) \otimes|0\rangle \\
\cdot \\
\cdot \\
\left.\cdot \cos \frac{g t}{\sqrt{2}}|1000\rangle-\sin \frac{g t}{\sqrt{2}} e^{i \theta}|0100\rangle\right)=\left(\cos \frac{g t}{\sqrt{2}}|1\rangle-\sin \frac{g t}{\sqrt{2}} e^{i \theta}|0\rangle\right) \otimes|1\rangle \otimes|0\rangle \otimes|0\rangle
\end{gathered}
$$
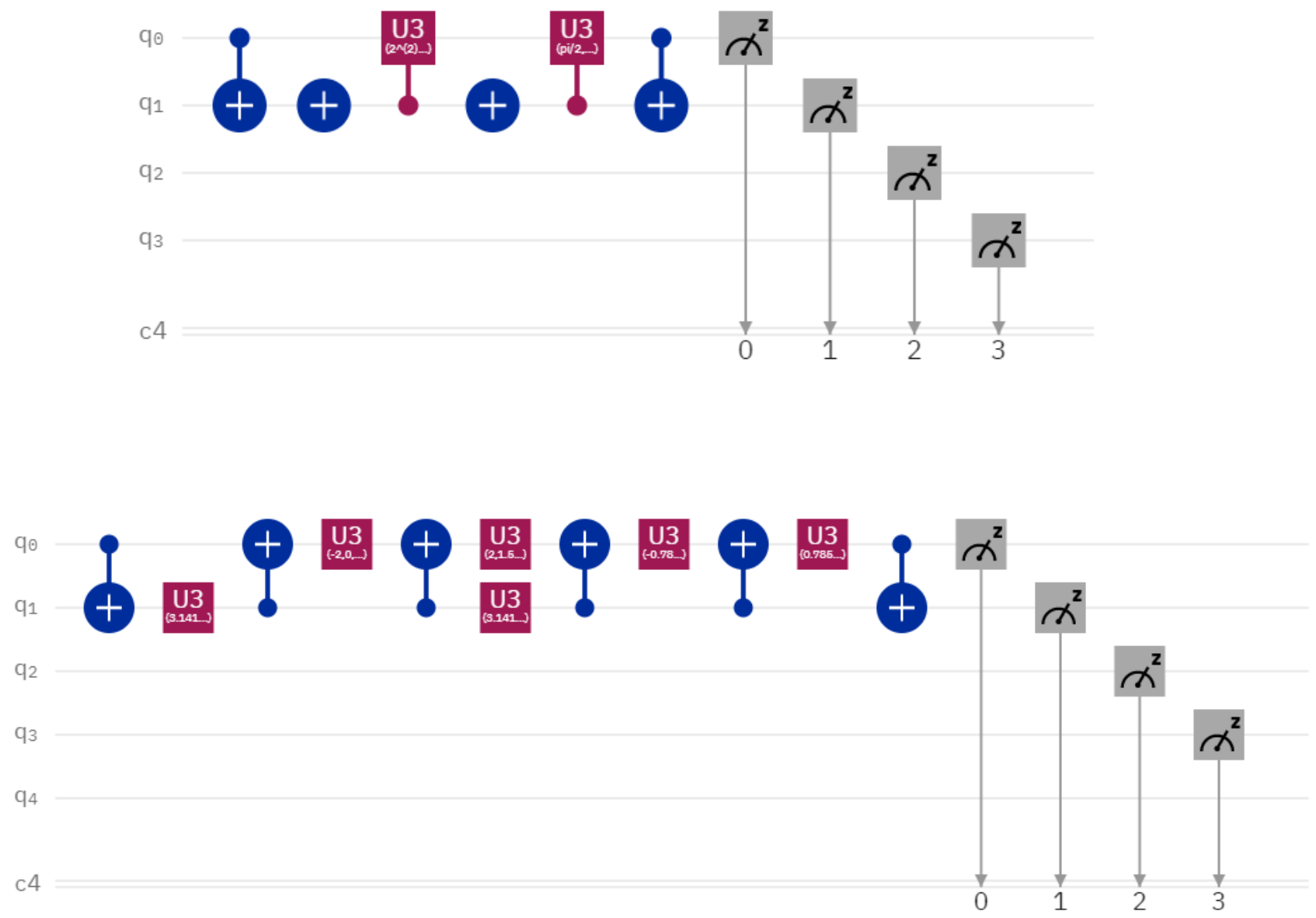

Figure 2: Filter for $|1000\rangle$ in case of $U_{y}(t)$ Operations (Upper image with cU3 gate and Lower image with Simplification of the circuit)

So, in the above segment, we computed the $U_{y}(t)$ operator and also disentangled the results. A filtered portion of our quantum circuit for the qubit state $|1000\rangle$ is shown above in Fig.(2). 
Now in order to compute $U_{x}(t)$ which is equal to $e^{-i X t}$, we first expand the expression using the Taylor expansion of the exponential function just like we did in earlier case as:

$$
\begin{gathered}
U_{x}(t)=\exp (-i t X)=\mathbb{I}+\sum_{m=1}^{\infty}(-i t)^{m} \frac{X^{m}}{m !} \\
\Longrightarrow U_{x}(t)=\mathbb{I}+(-i t)^{1} \frac{X}{1 !}+(-i t)^{2} \frac{X^{2}}{2 !}+(-i t)^{3} \frac{X^{3}}{3 !}+(-i t)^{4} \frac{X^{4}}{4 !}+(-i t)^{5} \frac{X^{5}}{5 !}+\ldots \ldots .
\end{gathered}
$$

Therefore by using the above equation, we can express $U_{x}(t)$ in terms of $e$ as:

\begin{tabular}{|l|l|}
\hline $\mathrm{U}_{\hat{x}}(t)[1,1]$ & $\exp \left(-\left(\frac{\omega_{0}}{2}\right) \mathrm{it}\right)$ \\
$\mathrm{U}_{\hat{x}}(t)[2,2]$ & $\exp (-(0) \mathrm{it})$ \\
$\mathrm{U}_{\hat{x}}(t)[3,3]$ & $\exp \left(\left(\frac{\omega_{0}}{2}\right) \mathrm{it}\right)$ \\
$\mathrm{U}_{\hat{x}}(t)[4,4]$ & $\exp \left(-\left(\omega+\frac{\omega_{0}}{2}\right) \mathrm{it}\right)$ \\
$\mathrm{U}_{\hat{x}}(t)[5,5]$ & $\exp (-(\omega) \mathrm{it})$ \\
$\mathrm{U}_{\hat{x}}(t)[6,6]$ & $\exp \left(-\left(\omega-\frac{\omega_{0}}{2}\right) \mathrm{it}\right)$ \\
$\mathrm{U}_{\hat{x}}(t)[7,7]$ & $\exp \left(-\left(\omega+\frac{\omega_{0}}{2}\right) \mathrm{it}\right)$ \\
$\mathrm{U}_{\hat{x}}(t)[8,8]$ & $\exp (-(\omega) \mathrm{it})$ \\
$\mathrm{U}_{\hat{x}}(t)[9,9]$ & $\exp \left(-\left(\omega-\frac{\omega_{0}}{2}\right) \mathrm{it}\right)$ \\
\hline
\end{tabular}

In case of $U_{x}(t)$ Operator also; we will consider a $16 \times 16$ matrix (in place of a $9 \times 9$ matrix) because of same reason mentioned before and also we will construct the matrix in the same pattern as mentioned in case of $U_{y}(t)$ operator. It is easy to observe as $\mathrm{X}$ is a diagonal matrix, each diagonal element of $U_{x}(t)$ makes an exact Taylor expansion of the exponential function

(**The $16 \times 16$ matrix for both $U_{y}(t)$ and $U_{x}(t)$ operators are mentioned in the next sub-section.)

Again, we operate this operator on different 4-qubits states (in our situation we need only nine of the sixteen 4-qubit states because for the other seven states we will get the same Unitary matrix as result.) and then study the results for the same given in Table(II):

\begin{tabular}{|c|l|}
\hline Qubit states & Results after $\mathrm{U}_{x}(t)$ acts \\
\hline$|0000\rangle$ & $\mathrm{e}^{\left(-\frac{\omega_{0}}{2}\right) i t}|0000\rangle$ \\
\hline$|0001\rangle$ & $\mathrm{e}^{(0) i t}|0000\rangle$ \\
\hline$|0010\rangle$ & $\mathrm{e}^{\left(\frac{\omega_{0}}{2}\right) i t}|0000\rangle$ \\
\hline$|0011\rangle$ & $\mathrm{e}^{-\left(\omega+\frac{\omega_{0}}{2}\right) i t}|0011\rangle$ \\
\hline$|0100\rangle$ & $\mathrm{e}^{(-\omega) i t}|0100\rangle$ \\
\hline$|0101\rangle$ & $\mathrm{e}^{-\left(\omega-\frac{\omega_{0}}{2}\right) i t}|0101\rangle$ \\
\hline$|0110\rangle$ & $\mathrm{e}^{-\left(\omega+\frac{\omega_{0}}{2}\right) i t}|0110\rangle$ \\
\hline$|0111\rangle$ & $\mathrm{e}^{(-\omega) i t}|0111\rangle$ \\
\hline$|1000\rangle$ & $\mathrm{e}^{-\left(\omega-\frac{\omega_{0}}{2}\right) i t}|1000\rangle$ \\
\hline
\end{tabular}

TABLE II. Operator $U_{x}(l)$ acting on Qubit States.

From the above table we can see the effect of $U_{x}(t)$ operator acting on the different 4-qubit states and we can construct the Quantum circuit for the same. A filtered portion of our quantum circuit for the qubit state $|0000\rangle$ is shown in Fig.(3) in the next page. 


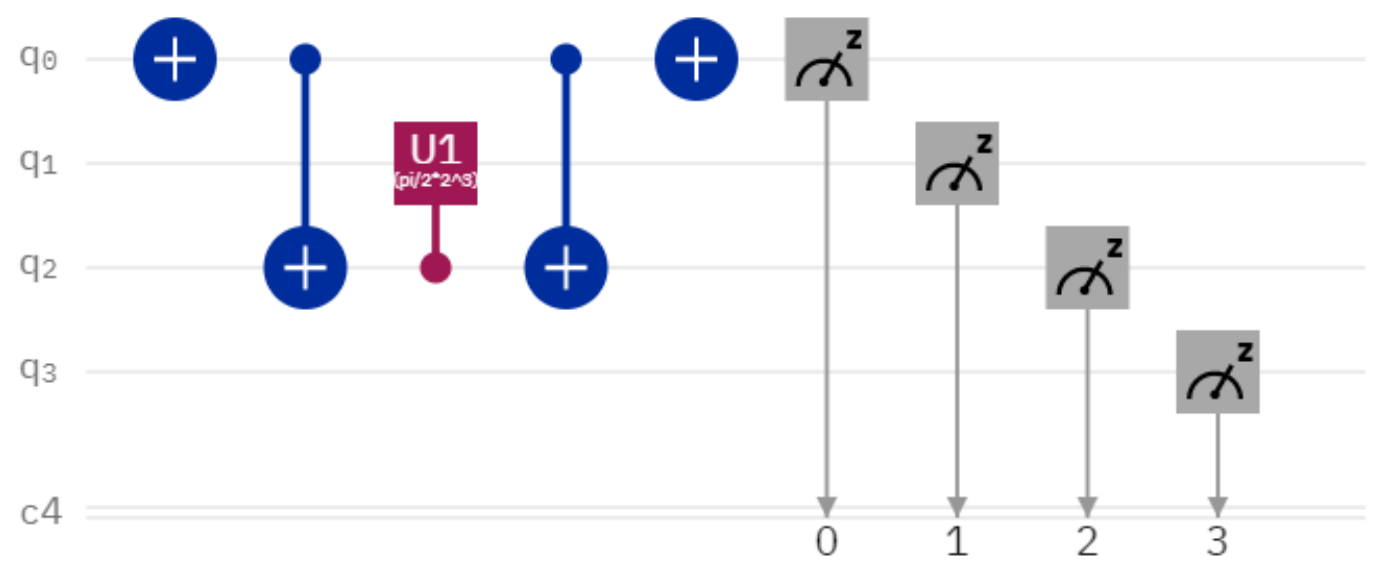

Figure 3: Filter for $|0000\rangle$ in case of $U_{x}(t)$ Operations

Now, we know how to implement both the parts of our Unitary operator and the complete unitary matrix $(16 \times 16)$ can be implemented by operating both the operations in series. In this way we can easily calculate our Unitary operators for Bosonic system and also simulate the Unitary Operators for a Quantum Harmonic Oscillator. We present its simulation results on IBM quantum computer in the form of graphs later on. Each simulation is carried on IBMQ-qasm Simulator using 8192 shots for better accuracy.

\section{UNITARY OPERATOR MATRIX REPRESENTATIONS}

The $16 \times 16$ Matrix representation of the Unitary operators $U_{y}(t)$ and $U_{x}(t)$ are:

$$
U_{y}(t)=\left[\begin{array}{cccccccccccccccc}
A & 0 & 0 & 0 & B & 0 & 0 & 0 & 0 & 0 & 0 & 0 & 0 & 0 & 0 & 0 \\
0 & A & 0 & B & 0 & B & 0 & 0 & 0 & 0 & 0 & 0 & 0 & 0 & 0 & 0 \\
0 & 0 & A & 0 & B & 0 & 0 & 0 & 0 & 0 & 0 & 0 & 0 & 0 & 0 & 0 \\
0 & C & 0 & A & 0 & 0 & 0 & B & 0 & 0 & 0 & 0 & 0 & 0 & 0 & 0 \\
C & 0 & C & 0 & A & 0 & B & 0 & B & 0 & 0 & 0 & 0 & 0 & 0 & 0 \\
0 & C & 0 & 0 & 0 & A & 0 & B & 0 & 0 & 0 & 0 & 0 & 0 & 0 & 0 \\
0 & 0 & 0 & 0 & C & 0 & A & 0 & 0 & 0 & 0 & 0 & 0 & 0 & 0 & 0 \\
0 & 0 & 0 & C & 0 & C & 0 & A & 0 & 0 & 0 & 0 & 0 & 0 & 0 & 0 \\
0 & 0 & 0 & 0 & C & 0 & 0 & 0 & A & 0 & 0 & 0 & 0 & 0 & 0 & 0 \\
0 & 0 & 0 & 0 & 0 & 0 & 0 & 0 & 0 & 1 & 0 & 0 & 0 & 0 & 0 & 0 \\
0 & 0 & 0 & 0 & 0 & 0 & 0 & 0 & 0 & 0 & 1 & 0 & 0 & 0 & 0 & 0 \\
0 & 0 & 0 & 0 & 0 & 0 & 0 & 0 & 0 & 0 & 0 & 1 & 0 & 0 & 0 & 0 \\
0 & 0 & 0 & 0 & 0 & 0 & 0 & 0 & 0 & 0 & 0 & 0 & 1 & 0 & 0 & 0 \\
0 & 0 & 0 & 0 & 0 & 0 & 0 & 0 & 0 & 0 & 0 & 0 & 0 & 1 & 0 & 0 \\
0 & 0 & 0 & 0 & 0 & 0 & 0 & 0 & 0 & 0 & 0 & 0 & 0 & 0 & 1 & 0 \\
0 & 0 & 0 & 0 & 0 & 0 & 0 & 0 & 0 & 0 & 0 & 0 & 0 & 0 & 0 & 1
\end{array}\right]
$$

Where;

$$
\mathrm{A}=\cos \left(\frac{g t}{\sqrt{2}}\right) ; \quad \mathrm{B}=-i \sin \left(\frac{g t}{\sqrt{2}}\right) e^{i \theta} \quad \text { and } \quad \mathrm{C}=-i \sin \left(\frac{g t}{\sqrt{2}}\right) e^{-i \theta}
$$




$$
U_{x}(t)=\left[\begin{array}{llllllllllllllll}
S & 0 & 0 & 0 & 0 & 0 & 0 & 0 & 0 & 0 & 0 & 0 & 0 & 0 & 0 & 0 \\
0 & 1 & 0 & 0 & 0 & 0 & 0 & 0 & 0 & 0 & 0 & 0 & 0 & 0 & 0 & 0 \\
0 & 0 & \frac{1}{S} & 0 & 0 & 0 & 0 & 0 & 0 & 0 & 0 & 0 & 0 & 0 & 0 & 0 \\
0 & 0 & 0 & P & 0 & 0 & 0 & 0 & 0 & 0 & 0 & 0 & 0 & 0 & 0 & 0 \\
0 & 0 & 0 & 0 & Q & 0 & 0 & 0 & 0 & 0 & 0 & 0 & 0 & 0 & 0 & 0 \\
0 & 0 & 0 & 0 & 0 & R & 0 & 0 & 0 & 0 & 0 & 0 & 0 & 0 & 0 & 0 \\
0 & 0 & 0 & 0 & 0 & 0 & P & 0 & 0 & 0 & 0 & 0 & 0 & 0 & 0 & 0 \\
0 & 0 & 0 & 0 & 0 & 0 & 0 & Q & 0 & 0 & 0 & 0 & 0 & 0 & 0 & 0 \\
0 & 0 & 0 & 0 & 0 & 0 & 0 & 0 & R & 0 & 0 & 0 & 0 & 0 & 0 & 0 \\
0 & 0 & 0 & 0 & 0 & 0 & 0 & 0 & 0 & 1 & 0 & 0 & 0 & 0 & 0 & 0 \\
0 & 0 & 0 & 0 & 0 & 0 & 0 & 0 & 0 & 0 & 1 & 0 & 0 & 0 & 0 & 0 \\
0 & 0 & 0 & 0 & 0 & 0 & 0 & 0 & 0 & 0 & 0 & 1 & 0 & 0 & 0 & 0 \\
0 & 0 & 0 & 0 & 0 & 0 & 0 & 0 & 0 & 0 & 0 & 0 & 1 & 0 & 0 & 0 \\
0 & 0 & 0 & 0 & 0 & 0 & 0 & 0 & 0 & 0 & 0 & 0 & 0 & 1 & 0 & 0 \\
0 & 0 & 0 & 0 & 0 & 0 & 0 & 0 & 0 & 0 & 0 & 0 & 0 & 0 & 1 & 0 \\
0 & 0 & 0 & 0 & 0 & 0 & 0 & 0 & 0 & 0 & 0 & 0 & 0 & 0 & 0 & 1
\end{array}\right]
$$

Where,

$$
\mathrm{P}=e^{-\left(\omega+\frac{\omega_{0}}{2}\right) i t} ; \quad \mathrm{Q}=e^{(-\omega) i t} ; \quad \mathrm{R}=e^{-\left(\omega-\frac{\omega_{0}}{2}\right) i t} ; \quad \mathrm{S}=e^{\left(-\frac{\omega_{0}}{2}\right) i t} \quad \text { and } \quad \frac{1}{\mathrm{~S}}=\frac{1}{e^{\left(-\frac{\omega_{0}}{2}\right) i t}}=e^{\left(\frac{\omega_{0}}{2}\right) i t}
$$

\section{RESULTS}

In our paper, we use the idea of Pauli Matrices Equivalents for Bosonic particles and see the implementation of the equivalent matrices. Then we introduce a coupled Quantum Harmonic Oscillator to the Bosonic system and try to implement its Unitary Operator to the system using our previous section's knowledge and also simulate the Unitary Operators using IBMQ-experience (in 8192 shots for better accuracy). The Results of the simulation are shown in Fig.(4), Fig.(5) and Fig.(6) respectively.

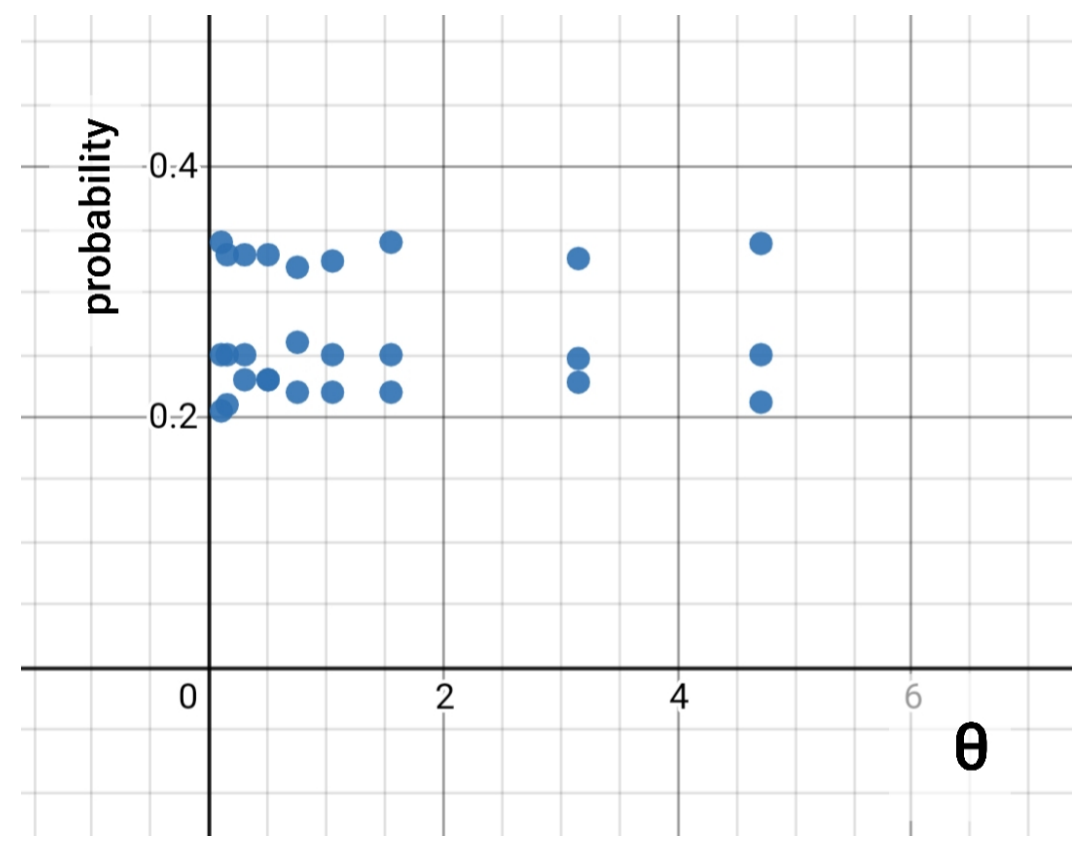

Figure 4: Graph between $\theta$ vs Probability 


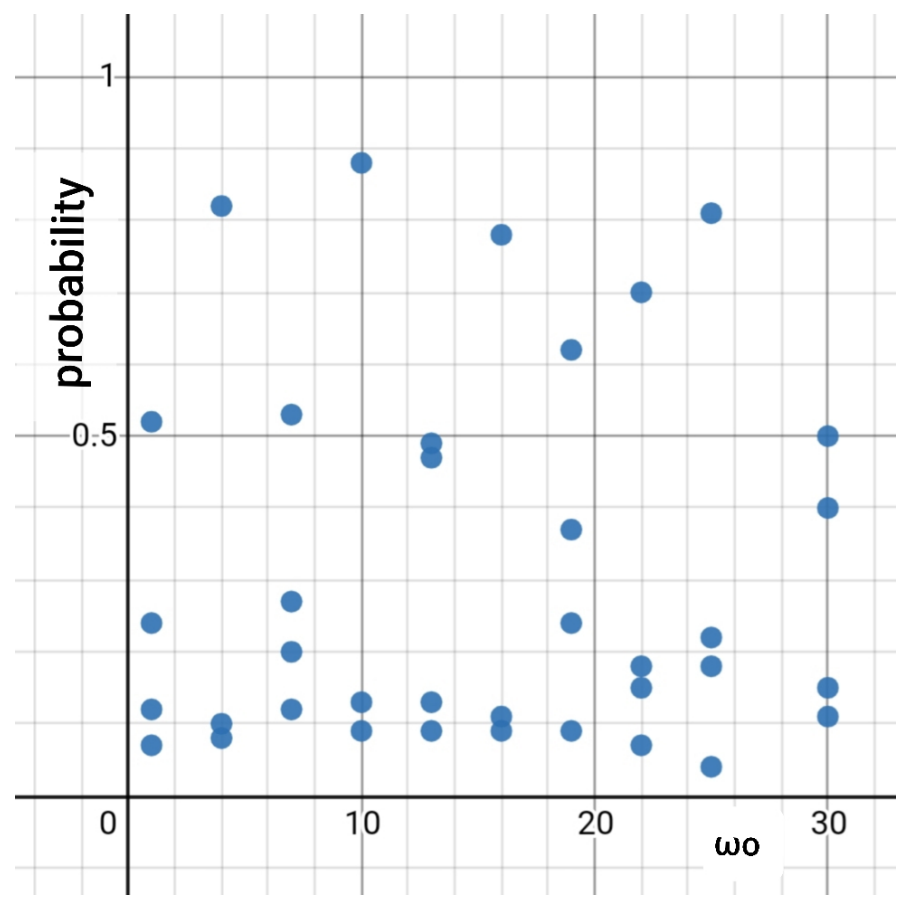

Figure 5: Graph between $\omega_{0}$ vs Probability

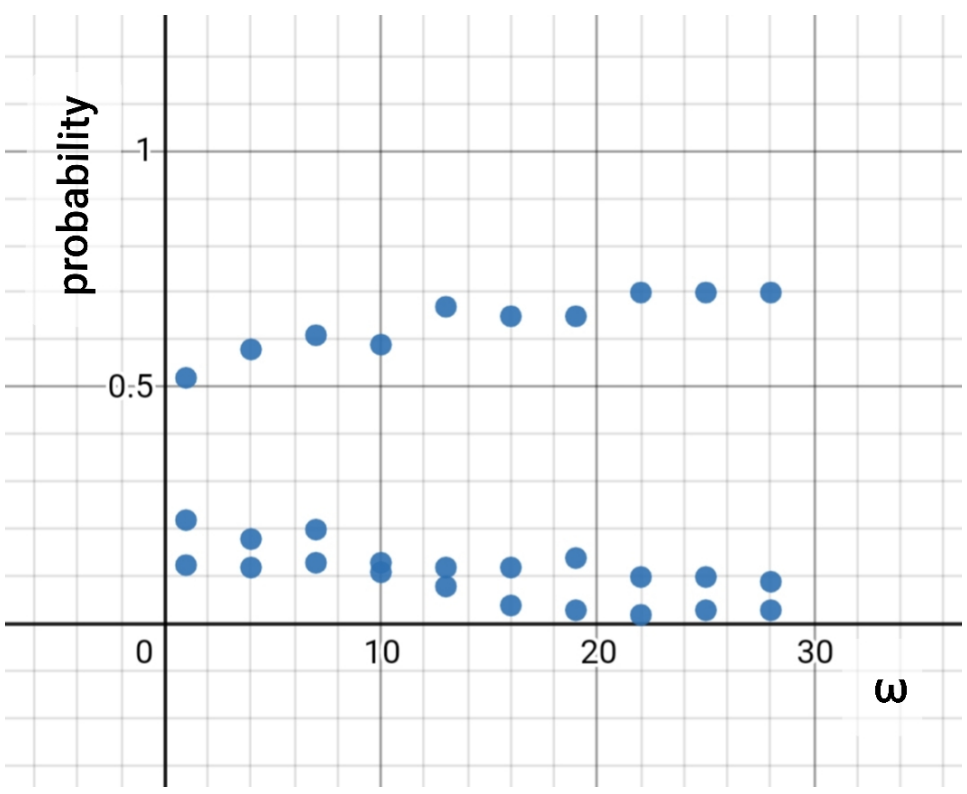

Figure 6: Graph between $\omega$ vs Probability

\section{CONCLUSION}

In this project, we visualized the process for simulating a Quantum Harmonic Oscillator (QHO), associated to a Bosonic system, using IBMQ-experience. In our case we derived the Unitary Operators for the (QHO) by using the Pauli Matrix equivalents for Bosonic system and after that we associated the usable Quantum States (4-qubit states) with the Unitary Operator (which is in-turn formed by combining the $U_{y}(t)$ and $U_{x}(t)$ Operators in series). From the above process we can infer that the Unitary Operator is the sole factor which 
is necessary for simulating the $(\mathrm{QHO})$ and we simulate the system by taking 8192 shots in IBMQ-experience because it will increase the effectiveness of our results and decrease the chance of any error in our simulation.

We conclude with one last important factor which we should consider while simulating the system and it is related to the disentanglement of the final Quantum states after the Unitary Operator acts on the initial different 4-qubit states associated with the system. For simulation purpose, it is very important that the final quantum states are completely disentangled so that we can construct the different quantum circuits which in-turn helps in simulation.

\section{ACKNOWLEDGEMENTS}

RT would like to thank Bikash K. Behera of Bikash's Quantum (OPC) Pvt. Ltd. and Prof. Prasanta K. Panigrahi of Indian Institute of Science Education and Research, Kolkata for providing him guidance in this project. He also acknowledge the support of IBM Quantum Experience for producing experimental results and the results as well as views expressed are solely those of the author and do not reflect the official policy or position of IBM or the IBM Quantum Experience team.

\section{References}

[1] Quantum Harmonic Oscillator, https://en.wikipedia.org/wiki/Quantum_harmonic_oscillator

[2] Quantum Harmonic Oscillator Diagram, https://www.sciencedirect.com/science/article/abs/ pii/S1568494618302667

[3] Uncertainty Principle, https://en.wikipedia.org/wiki/Uncertainty_principle

[4] Quantum Harmonic Oscillator, https://phys.libretexts.org/Bookshelves/University_ Physics/Book\%3A_University_Physics_(OpenStax)/Map\%3A_University_Physics_III_-_Optics_ and_Modern_Physics_(OpenStax)/07\%3A_Quantum_Mechanics/7.06\%3A_The_Quantum_Harmonic_ Oscillator

[5] C. Bayindir, Rogue Quantum Harmonic Oscillator, arXiv:1902.08823 [quant-ph](2019)

[6] Y. Rezek, The Quantum Harmonic Oscillator as a Thermodynamic Engine (2004).

[7] Rajdeep Tah. Calculating the Pauli Matrix equivalent for Spin-1 Particles and further implementingit to calculate the Unitary Operators of the Harmonic Oscillator involving a Spin-1 System. 2020.hal02909703, URL: https://hal . archives-ouvertes. fr/hal-02909703

[8] D. J. Griffths, Introduction to Quantum Mechanics, Pearson Prentice Hall (2004).

[9] Relation between Hamiltonian and the Operators, URL: https://en . wikipedia .org/wiki/Creation_ and_annihilation_operators

[10] Quantum Fourier Transform, URL: https://en.wikipedia.org/wiki/Quantum_Fourier_transform

[11] V. K. Jain, B. K. Behera, and P. K. Panigrahi, Quantum Simulation of Discretized Harmonic Oscillator on IBMQuantum Computer, DOI: 10.13140/RG.2.2.26280.93448(2019)

[12] Jaynes-Cummings model'URL: https://en.wikipedia.org/wiki/Jaynes\%E2\%80\%93Cummings_ model

[13] S. Agarwal, S. M. H. Rafsanjani and J. H. Eberly, Tavis-Cummings model beyond the rotating wave approximation: Quasi-degenerate qubits, Phys. Rev. A 85, 043815 (2012).

[14] B. Militello, H. Nakazato, and A. Napoli1, 2 : Synchronizing Quantum Harmonic Oscillators through Two-Level Systems, Phys. Rev. A 96, 023862 (2017).

[15] Holstein-Primakoff transformation, URL: https://en.wikipedia.org/wiki/Holstein\%E2\%80\% 93Primakoff_transformation 